\title{
Joint CS-MRI Reconstruction and Segmentation with a Unified Deep Network
}

\author{
Liyan Sun ${ }^{\dagger}$, Zhiwen Fan ${ }^{\dagger}$, Yue Huang, Xinghao Ding ${ }^{\star}$, John Paisley ${ }^{\ddagger}$ \\ Fujian Key Laboratory of Sensing and Computing for Smart City, Xiamen University, Fujian, China \\ ${ }^{\dagger}$ The co-first authors contributed equally. \\ ${ }^{\star}$ Correspondence: dxh@xmu.edu.cn. \\ ${ }^{\ddagger}$ Department of Electrical Engineering, Columbia University, New York, NY, USA
}

\begin{abstract}
The need for fast acquisition and automatic analysis of MRI data is growing in the age of big data. Although compressed sensing magnetic resonance imaging (CS-MRI) has been studied to accelerate MRI by reducing $\mathrm{k}$-space measurements, in current CS-MRI techniques MRI applications such as segmentation are overlooked when doing image reconstruction. In this paper, we test the utility of CS-MRI methods in automatic segmentation models and propose a unified deep neural network architecture called SegNetMRI which we apply to the combined CS-MRI reconstruction and segmentation problem. SegNetMRI is built upon a MRI reconstruction network with multiple cascaded blocks each containing an encoder-decoder unit and a data fidelity unit, and MRI segmentation networks having the same encoder-decoder structure. The two subnetworks are pre-trained and finetuned with shared reconstruction encoders. The outputs are merged into the final segmentation. Our experiments show that SegNetMRI can improve both the reconstruction and segmentation performance when using compressive measurements.
\end{abstract}

\section{Introduction}

Magnetic resonance imaging (MRI) is an important technique for visualizing human tissue. The raw measurements come in the form of Fourier transform coefficients in "k-space" and the MRI can be viewed after an inverse 2D Fourier transform of the fully sampled k-space. Conventionally, radiologists view MRI for diagnosis. However, in areas where medical expertise may be lacking or not sufficient to meet demand, automated methods may also be useful. To this end, automatic MR image segmentation is essential because it allows for finer localization of focus. To take brain segmentation for example, usually four structures emerge including background, gray matter (GM), white matter (WM) and cerebrospinal fluid (CSF). Lesions appearing in white matter are closely associated with various issues such as strokes and Alzheimer's disease [Steenwijk et al., 2013].

Although rich anatomical information can be provided by MRI, it is limited by a long imaging period. This can in- troduce motion artifacts caused by movement of the patient [Atkinson et al., 1999] or induce psychological pressures brought by claustrophobia [Hricak and Amparo, 1984]. Thus accelerating imaging speed while maintaining high imaging quality is key for MRI. Compressed sensing (CS) theory [Donoho, 2006; Candès et al., 2006], which shows the possibility of recovering signals with sub-Nyquist sampling rates, has been introduced to the field of MRI to accelerate imaging. In 2017, the US Food and Drug Administration (FDA) approved CS-MRI techniques for use by two major MRI vendors: Siemens and GE [Fessler, 2017]. Thus, one can expect increasing deployment of CS-MRI technique in the future for real-world applications.

To our knowledge, current segmentation algorithms for MRI assume a "clean" (i.e., fully-sampled) image as input and do not take CS-MRI scenarios into consideration. Likewise, CS-MRI reconstruction methods do not consider their output's potential downstream segmentation. Although experienced human experts can make relatively robust decisions with CS-reconstructed images, the anticipated increase in the number of CS-reconstructed MRI for clinical application will call for automatic segmentation algorithms optimized for this type of data. Therefore, a unified approach to MRI reconstruction/segmentation under the compressed sensing framework is worthy of exploration.

In this paper, we develop a unified deep neural network called SegNetMRI for joint MRI reconstruction and segmentation with compressive measurements. We build SegNetMRI on two networks: an MRI reconstruction network (MRN) and MRI segmentation network (MSN). The MSN is an encoderdecoder structure network and SegNetMRI is made up of basic blocks which consists of encoder-decoder and data fidelity units. The MRN is pre-trained with pairs of artificially undersampled and their corresponding fully-sampled MRI and the MSN with fully-sampled MRI and corresponding segmentation labels. We fine-tune the resulting unified network with MSN and MRN sharing the encoder component. With the basic features produced by the fine-tuned encoder, the MRI reconstruction and segmentation can regularize each other this way. The outputs are merged by $1 \times 1$ convolution.

\section{Background and Related Work}

MRI segmentation Broadly speaking, the research in MRI segmentation can be categorized into three classes: (1) atlas- 
based segmentation with registration; (2) machine learning models with hand-crafted features; (3) deep learning models. Atlas-based segmentation AAljabar et al., 2009, Artaechevarria et al., 2009] requires accurate registration and is time-consuming, so it is impractical in real applications that require fast speed. In the second class, manually designed features are fed into classifiers, e.g., 3D Haar/spatial features into random forests |Wang et al., 2015|. These hand-crafted features are not very flexible in encode diverse patterns within MRI data. Recently deep learning based models have been propose, $\mathrm{d}$ such as a 2D convolutional neural network [Nie et al., 2016; Moeskops et al., 2016], a 3D convolutional neural network [Çiçek et al., 2016, Chen et al., 2017], and parallelized long short-term memory (LSTM) [Stollenga et al., 2015]. They can learn semantic image features from data, leading to the state-of-the-art performance in MRI segmentation. However, these MRI segmentation models do not take the input quality into consideration, but assume full measurements.

Compressed Sensing MRI We denote the underlying vectorized MRI $x \in \mathbb{C}^{P \times 1}$ which we seek to reconstruct from the sub-sampled vectorized k-space data $y \in \mathbb{C}^{Q \times 1}(Q \ll P)$. CS-MRI is then typically formulated as

$$
x=\arg \min \left\|F_{u} x-y\right\|_{2}^{2}+f_{\theta}(x),
$$

where the $F_{u} \in \mathbb{C}^{Q \times P}$ denotes the under-sampled Fourier matrix. The $\ell_{2}$ term is the data fidelity and $f_{\theta}(\cdot)$ represents a regularization with parameter $\theta$ to constrain the solution space.

The main research focus of CS-MRI is proposing better $f_{\theta}$ and efficient optimization techniques. In the first CS-MRI work called SparseMRI [Lustig et al., 2007], wavelet domain $\ell_{1}$ sparsity plus image total variation are imposed as regularizations. More complicated wavelet variants are designed for CS-MRI in PANO [Qu et al., 2014] and GBRWT [Lai et al., 2016]. Dictionary learning techniques are also introduced in CS-MRI, e.g., DLMRI [Ravishankar and Bresler, 2011] and BPTV |Huang et al., 2014]. These works can all be categorized as sparsity-based CS-MRI methods; they model the MRI with a "shallow" sparse prior, which often tends to oversmooth the image.

Recently, deep neural networks have been introduced to CS-MRI. Researchers have directly applied convolutional neural networks (CNN) to learn a direct mapping from the zero-filled MRI $F_{u}^{H} y$ (obtained by zero-padding the unsampled positions in k-space) to the true MRI [Wang et al., 2016]. A deep residual architecture was also proposed for this same mapping [Lee et al., 2017]. Data fidelity terms have been incorporated into the deep neural network by [Schlemper et al., 2017] to add more guidance. These deep learning based CSMRI models have achieved higher reconstruction quality and faster reconstruction speed.

Combining visual tasks The combination of different visual tasks in a unified model has been investigated in the field of computer vision. For example, a joint blind image restoration and recognition model [Zhang et al., 2011] based sparse

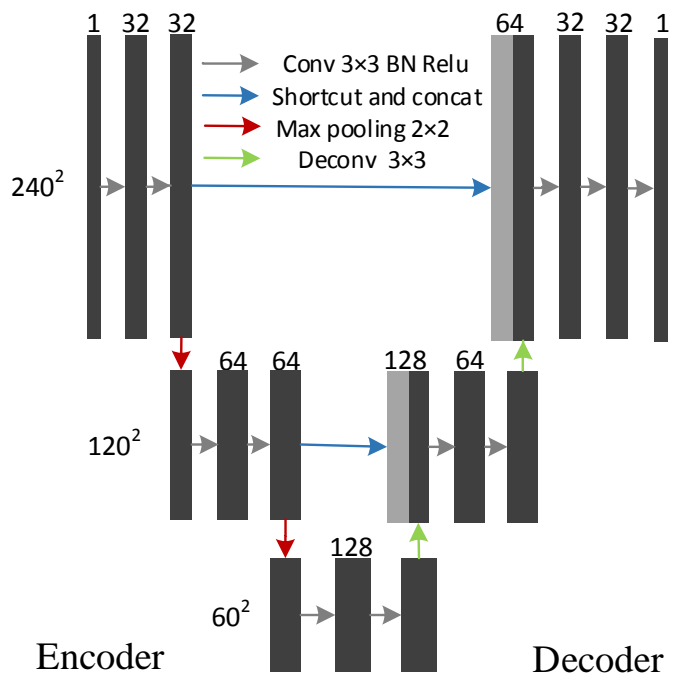

Figure 1: The MSN architecture composed of an encoder and decoder. It is used to assess the segmentation accuracy on different reconstructed MRI produced by various CS-MRI methods.

coding was proposed for face recognition with low-quality images. In the image dehazing model AOD-Net [Li et al., 2017], the researchers discuss detection in the presence of haze by performing dehazing during detection. In the MRI field, 3T-obtained images have been used in joint segmentation and super-resolution (7T) image generation [Bahrami et al., 2017.

\section{Methodology}

In this section, we give a detailed description of the proposed SegNetMRI model. First, we propose a segmentation network baseline and test popular CS-MRI methods on the well-trained model. Next we propose a MRI reconstruction network formed by cascading basic blocks. We show the proposed MRI reconstruction network achieves better performance on segmentation over conventional sparsity based CSMRI models, but still inferior to the full-sampled MR image. We further propose the SegNetMRI model to merge the MRI reconstruction and segmentation into an single model.

\subsection{Illustration: Segmentation after CS-MRI}

We first test several popular CS-MRI outputs on an automatic MRI segmentation model to assess the impact of compressed sensing for this task. Inspired by the state-of-the-art medical image segmentation model U-Net [Ronneberger et al., 2015], we propose the MRI segmentation network (MSN) shown in Figure 1. The segmentation encoder (SE) component, using convolution and pooling, can extract features from the input image at different scales, and the segmentation decoder (SD) component, using a deconvolution operation, predicts the the pixel-wise segmentation class from these features. Shortcut 


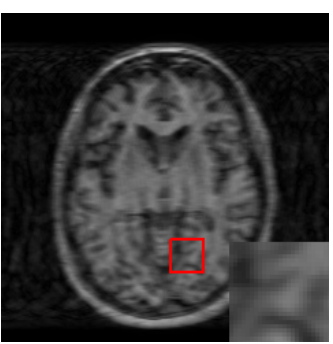

(a) ZF

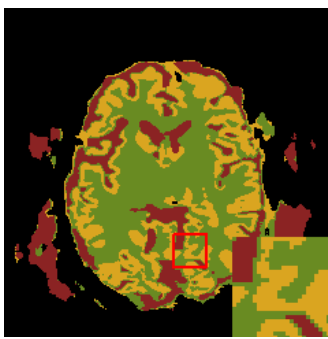

(f) ZF Seg

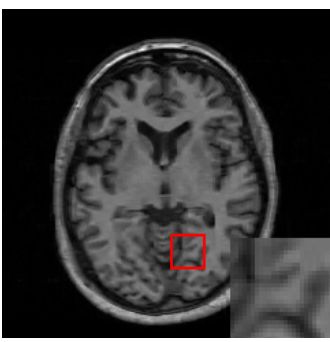

(b) PANO

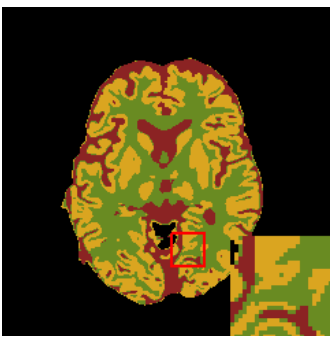

(g) PANO Seg

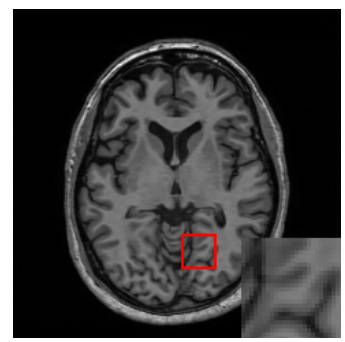

(c) $\mathrm{MRN}_{5}$

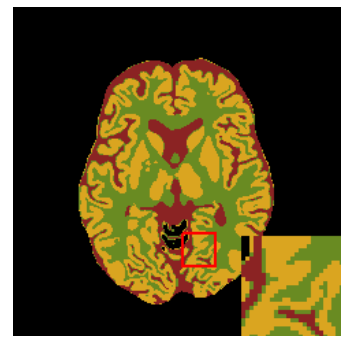

(h) $\mathrm{MRN}_{5} \mathrm{Seg}$

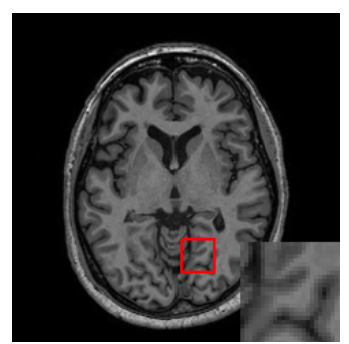

(d) Full-sample (GT)

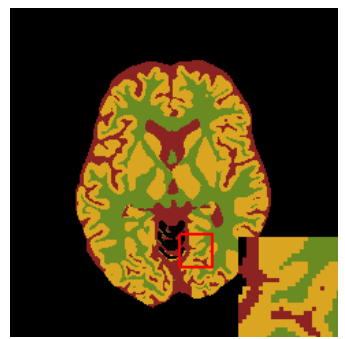

(i) Full-sample Seg

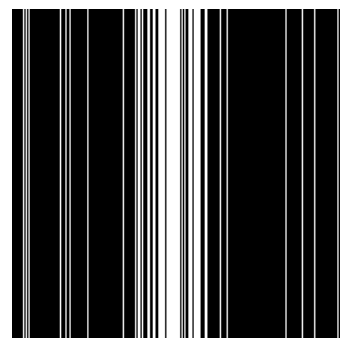

(e) Mask

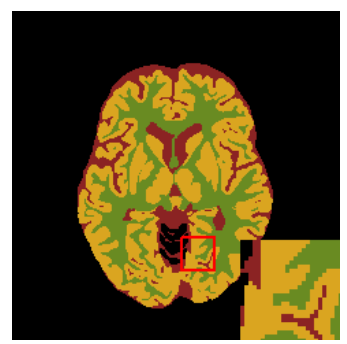

(j) Manual Seg

Figure 2: First row: The reconstructed MRI using different CS-MRI methods and a $20 \%$ sampling mask. These are then segmented by an independently-trained segmentation model based on the state-of-the-art U-Net (referred to as MSN in this paper for its MRI application).

connections are used by this model to directly send lowerlayer features to high-layer features by concatenation.

Training this MSN model using fully-sampled MRI and their segmentation labels, we test this models performance on reconstructed MRI produced by various CS-MRI methods. We use a $20 \%$ Cartesian under-sampling mask as shown in Figure 2(e) Our tested methods including the degraded zero-filled (ZF) reconstruction as baseline, PANO [Qu et al., 2014] and the proposed MRN model which will be discussed in the following section 1 .

We observe that the zero-filled (ZF) reconstruction in Figure 2(a) produces a low-quality MR image, which leads to the poor segmentation performance shown in Figure 2(f) The PANO reconstructed MRI in Figure 2(b) has been segmented better in Figure 2(g), but is still far from satisfactory because of the loss of structural details in the reconstruction. The segmentation using the fully-sampled (FS) MRI in Figure 2(d) is shown in Figure 2(i) . Though this isn't the ground truth segmentation, it is the segmentation performed on the ground truth MRI, and so represented an upper bound for CS-MRI on this segmentation task. The manually label segmentation is shown in Figure 2(j) This experiment shows that while CS-MRI can substantially improve the reconstruction quality visually, the fine structural details which are important for segmentation can still be mission, leaving much space for further improvement.

\subsection{An MRI Reconstruction Network}

Deep learning for CS-MRI has the advantage of large modeling capacity, fast running speed, and high-level semantic modeling ability, which eases the integration of high-

\footnotetext{
${ }^{1}$ We adjust the parameters of PANO for this problem.
}

level task information compared with traditional sparsitybased CS-MRI models. Therefore, we adopt the same MSN encoder-decoder architecture from Figure 1 as a basic encoder-decoder unit with a global residual shortcut, which has been proven to help network training. The encoderdecoder unit produces the reconstructed MRI. Since with deep neural networks the information loss can become severe, we introduce a data fidelity (DF) unit to help correct the Fourier coefficients of the reconstructed MRI produced by the encoder-decoder architecture on the sampled positions in k-space. This takes advantage of the fact that we have accurate measurements at the under-sampled k-space locations, and so the layers of the network should not override this information. (The details of the data fidelity unit can be found in [Schlemper et al., 2017].)

The encoder-decoder architecture and data fidelity unit make up a basic block. As more blocks are stacked in a cascaded manner, the quality of the reconstructed MRI of each block can be gradually improved [Schlemper et al., 2017]. We therefore cascade $N$ such basic units to form the MRI reconstruction network $\left(\mathrm{MRN}_{N}\right)$ in Figure 3. The reconstruction encoder in different blocks extract features at different depth. Previously in Figure 11, we observed that $\mathrm{MRN}_{5}$ achieves better reconstruction performance in Figure 2(c) than the non-deep PANO method, but the segmentation output in Figure 2(h) (MRN $\rightarrow$ MSN) is still inferior to the fully-sampled segmentation. This motivates the following joint framework.

\subsection{SegNetMRI: A Unified Deep Neural Network}

Based on these observations, we propose a joint framework for CS-MRI reconstruction/segmentation that uses a deep neural network. We call this joint network SegNetMRI, 


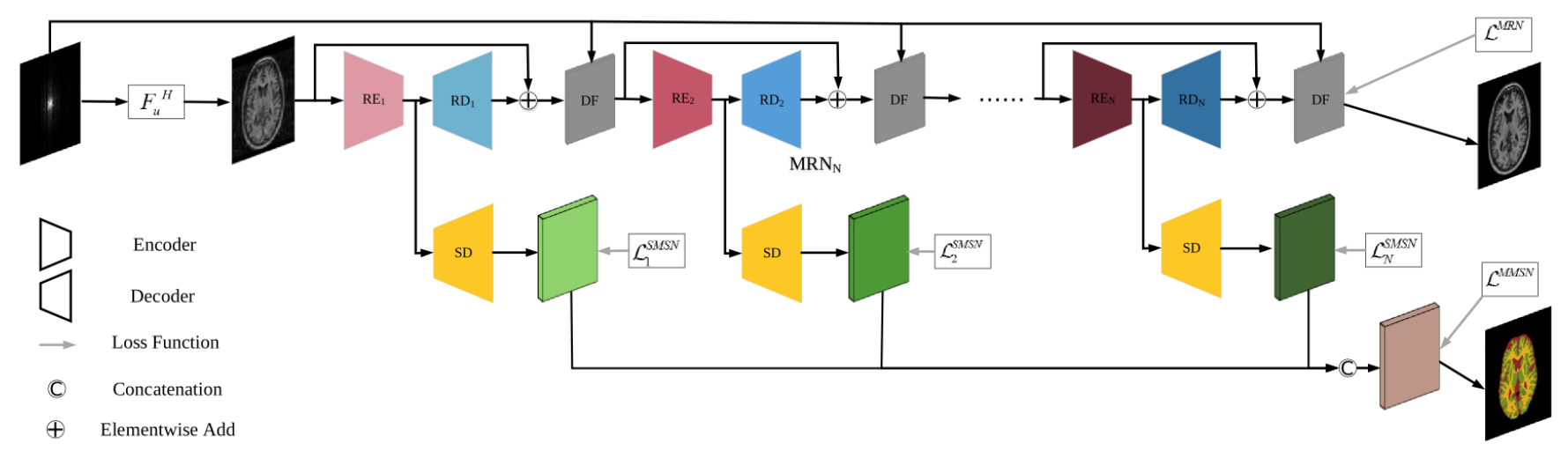

Figure 3: The SegNetMRI structure, formed by connecting the discussed MRN (top) for reconstruction, with MSN (bottom) for segmentation.

which is shown in Figure 3

To learn this model, we first pre-train separate models. We pre-train the $\mathrm{MRN}_{N}$ with under-sampled and fully-sampled MRI training pairs. Similarly, we pre-train the MSN with fully-sampled MRI and their corresponding segmentation labels. After training MSN, we leave out the encoder component (SE) and keep the decoder component (SD). We then connect the single decoder component of the MSN (SD) to each of the encoder components of each MRN (called $\mathrm{RE}_{n}$ ) within each block. The resulting $N$ outputs of the MSN decoder for each block are concatenated and merged to give the final segmentation via a $1 \times 1$ convolution. After pre-training separately and initializing the remaining parts, the parameters of $\mathrm{SegNetMRI}_{N}$ (with $N$ blocks in the MRN portion, but a single segmentation decoder duplicated $N$ times) are then fine-tuned. Therefore, both the reconstruction and segmentation tasks share the same encoders, but have separate decoders for their respective tasks.

The rationale for this architecture is the following:

1. With the pre-training of $\mathrm{MRN}_{N}$, the reconstruction encoder extracts basic features in different blocks. In SegNetMRI, the sharing of the reconstruction encoders between MRN and MSN means that these same features are used for both reconstruction and segmentation, which can help the two problems regularize each other.

2. The segmentation component uses information at various depths in the cascaded MRN, and combines this information in the decoder. The $1 \times 1$ convolution used to merge the outputs of the segmentation decoder at each layer can be viewed as ensemble learning.

Loss function We adopt the $\ell_{2}$ Euclidean distance as the loss function for pre-training the MRN,

$$
\mathcal{L}^{\mathrm{MRN}}=\frac{1}{L} \sum_{i=1}^{L}\left\|x_{i}^{f s}-x_{i}\right\|_{2}^{2},
$$

where $x_{i}^{f s}$ and $x_{i}$ are the $i^{\text {th }}$ full-sampled training image and the output of the MRN, respectively. To pre-train the MSN, we adopt the pixel-wise cross-entropy loss function

$$
\mathcal{L}^{\mathrm{MSN}}=-\sum_{i=1}^{L} \sum_{j=1}^{N} \sum_{c=1}^{C} t_{i j c}^{g t} \ln t_{i j c}
$$

where $C$ tissue classes are to be classified. $t^{g t}$ and $t$ is the pixel-wise ground-truth label and the MSN predicted label, respectively.

Pre-training the MRN and MSN this way, we then construct and fine-tune SegNetMRI with the following loss function

$$
\mathcal{L}^{\text {SegNetMRI }}=\mathcal{L}^{\mathrm{MRN}}+\lambda \mathcal{L}^{\mathrm{OMSN}}
$$

We set $\lambda=0.01$ in our experiments. Note the overall MSN (OMSN) loss containing $N+1$ loss function terms if the SegNetMRI contains $N$ blocks is

$$
\mathcal{L}^{\mathrm{OMSN}}=\frac{1}{N+1}\left(\mathcal{L}^{\mathrm{MMSN}}+\sum_{i=1}^{N} \mathcal{L}_{i}^{\mathrm{SMSN}}\right),
$$

where the $\mathcal{L}^{\mathrm{MMSN}}$ is the loss for the merged prediction and $\mathcal{L}^{\text {SMSN }}$ is the loss for each sub-MSN decoder prediction.

\section{Experiments and Discussions}

\subsection{Implementation Details}

Setup We implement all deep models on TensorFlow for the Python environment using a NVIDIA Geforce GTX 1080Ti with 11GB GPU memory and Intel Xeon CPU E52683 at $2.00 \mathrm{GHz}$. We show the hyperparameter settings of encoder-decoder architecture used for both MRN and MSN in Figure 1. We use batch normalization to stabilize training. ReLU is used as activation function except for the last convolution layer of the encoder-decoder unit within each MRN block, where the identity map is applied for residual learning. We apply Xavier initialization for pre-training MRN and MSN. MSN is pre-trained for 60,000 iterations using $64 \times 64$ fully-sampled MRI patches randomly cropped (16 patches in a batch) and MRN is pre-trained for 30,000 iterations using the entire training image (4 images in a batch). We then finetune the SegNetMRI model for 8, 000 further iterations using entire images ( 4 images in a batch). ADAM is chosen as optimizer. We select the initial learning rate to be 0.0005 , the first-order momentum to be 0.9 and the second momentum to be 0.999 .

Data We test our SegNetMRI architecture on the MRBrainS datasets from the Grand Challenge on MR Brain Image Segmentation (MRBrainS) Workshop |Mendrik et al., 


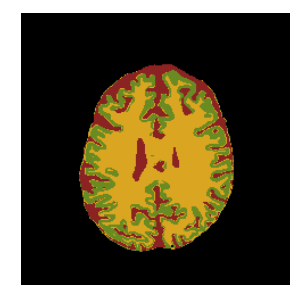

(a) GBRWT+MSN

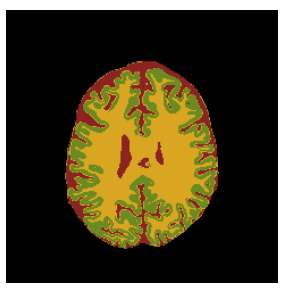

(b) $\mathrm{MRN}_{5}+\mathrm{MSN}$

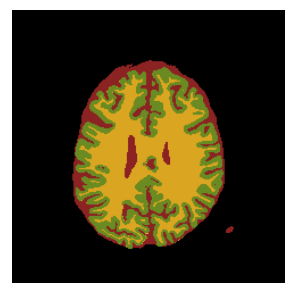

(c) |Liu et al., 2017|

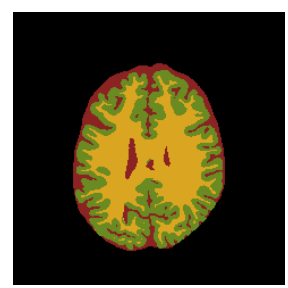

(d) $\mathrm{SegNetMRI}_{5}$

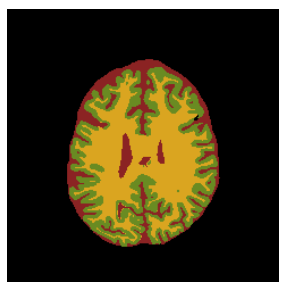

(e) FS+MSN

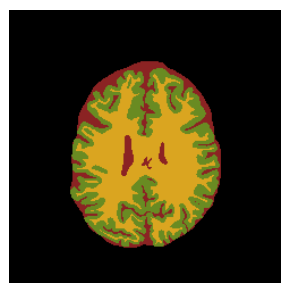

(f) GroundTruth

Figure 4: The segmentations of the compared methods.

\begin{tabular}{|l|c|c|c|c|c|c|c|c|c|}
\hline \multirow{2}{*}{ Methods } & \multicolumn{3}{|c|}{ GM } & \multicolumn{3}{c|}{ WM } & \multicolumn{3}{c|}{ CSF } \\
\cline { 2 - 11 } & DC & HD & AVD & DC & HD & AVD & DC & HD & AVD \\
\hline GBRWT+MSN $^{75.55}$ & 2.24 & 4.21 & 65.56 & 1.90 & 3.10 & 76.50 & 1.77 & 2.69 \\
\hline MRN $_{5}+$ MSN & 79.36 & 2.06 & 3.57 & 65.76 & 1.88 & 2.96 & 78.43 & 1.64 & 2.33 \\
\hline Liu et al., 2017 & 83.41 & 1.81 & 2.96 & 78.05 & 1.24 & 1.61 & 77.81 & 1.76 & 2.58 \\
\hline SegNetMRI & $\mathbf{8 6 . 3 8}$ & $\mathbf{1 . 6 6}$ & $\mathbf{2 . 5 2}$ & $\mathbf{8 1 . 4 9}$ & $\mathbf{1 . 0 8}$ & $\mathbf{1 . 3 4}$ & $\mathbf{7 9 . 2 3}$ & $\mathbf{1 . 6 1}$ & $\mathbf{2 . 2 3}$ \\
\hline FS+MSN & 87.36 & 1.60 & 2.33 & 85.94 & 1.00 & 1.14 & 81.01 & 1.61 & 2.18 \\
\hline
\end{tabular}

Table 1: The segmentation comparison of the different models using DC (\%), HD and AVD (\%) as metrics. FS+MSN is the segmentation performance when the ground truth MRI is known.

2015. The datasets are acquired using 3T MRI scans. Five datasets are provided containing T1-1mm, T1, T1-IR and T2FLAIR imaging modalities already registered and with manual segmentations. Here we use the T1 MRI data of the size $240 \times 240$ throughout the paper. We use four datasets for training (total 192 slices) and one dataset for testing (total 48 slices). We adopt the same data augmentation technique discussed in [Dong et al., 2017].

\subsection{Experimental Results}

To evaluate the segmentation performance, we compare the proposed $\mathrm{SegNetMRI}_{5}$ (5 blocks) with the inputting a fully-sampled MRI in MSN (FS+MSN) as ground truth performance, inputing the $\mathrm{MRN}_{5}$ reconstruction in MSN $\left(\mathrm{MRN}_{5}+\mathrm{MSN}\right)$ - i.e., no fine-tuning), inputting GBRWT reconstruction in MSN (GBRWT+MSN). GBRWT [Lai et al., 2016] represents the state-of-the-art performance in the conventional sparse based CS-MRI methods. Finally, we also compare with the joint framework proposed by [Liu et al., 2017], where only the reconstruction network is fine-tuned in $\mathrm{MRN}_{5}+\mathrm{MSN}$ using the loss function $\mathcal{L}^{\mathrm{MRN}}+\lambda \mathcal{L}^{\mathrm{MSN}}$. The same under-sampling mask shown in Figure 2(e) is again used.

We show qualitative comparison in Figure 4 We colorize segmentation corresponding white matter, gray matter and cerebrospinal fluid with yellow, green and red. We observe that the proposed $\mathrm{SegNetMRI}_{5}$ model provides better segmentation and approximates the ideal FS+MSN most closely, both of which are not perfect compared with the human labeling. For quantitative evaluation, we use the Dice Coefficient (DC), the 95th-percentile of the Hausdoff distance (HD) and the absolute volume difference (AVD), as also used in the MRBrainS challenge [Mendrik et al., 2015]. Larger DC, and smaller HD and AVD, indicate better segmentation. We show these results in Table 1 , which is consistent with the subjec-

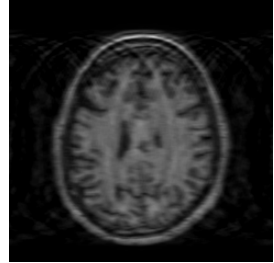

(a) ZF

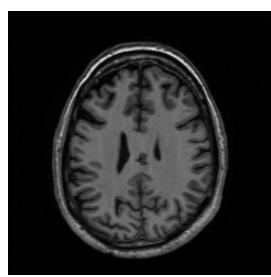

(d) Huang

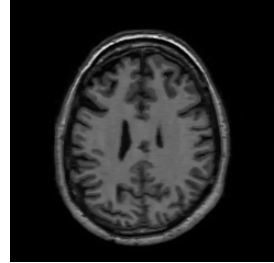

(b) GBRWT

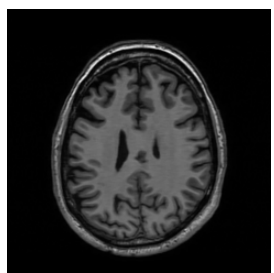

(e) $\mathrm{SegNetMRI}_{5}$

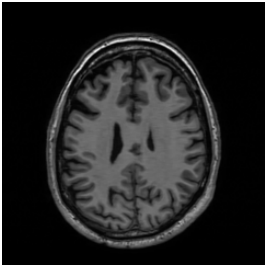

(c) $\mathrm{MRN}_{5}$

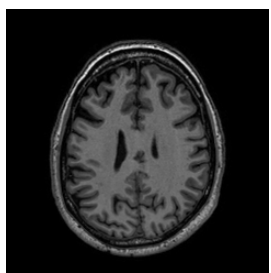

(f) FS

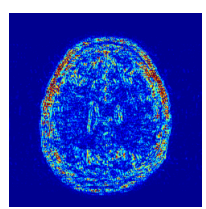

(g) GBRWT Error

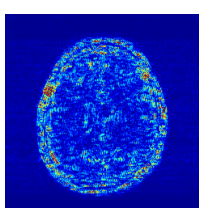

(h) $\mathrm{MRN}_{5}$ Error

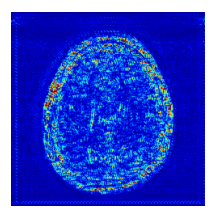

(i) Huang Error

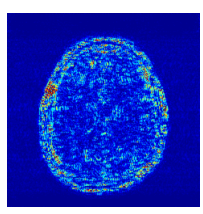

(j) SegNetMRI 5 Error
Figure 5: The reconstructed MR images with different CS-MRI methods in the first two rows and the residual maps in the third row.

tive evaluation.

In addition to the improvement of segmentation accuracy, we also evaluate the reconstruction quality of the SegNetMRI model. We show the reconstructed MRI from SegNetMRI the model in [Liu et al., 2017], $\mathrm{MRN}_{5}$ and GBRWT in Fig- 


\begin{tabular}{|l|c|c||c|c|}
\hline & GBRWT & MRN $_{5}$ & Liu et al., 2017 & SegNetMRI $_{5}$ \\
\hline PSNR & 31.80 & 33.94 & 33.47 & $\mathbf{3 4 . 2 7}$ \\
\hline NMSE & 0.0584 & 0.0361 & 0.0388 & $\mathbf{0 . 0 3 3 3}$ \\
\hline
\end{tabular}

Table 2: The averaged PSNR (dB) and NMSE on 37 test MRI data.

\begin{tabular}{|l|c|c|c|c|c|c|}
\hline GM & $\mathrm{B}_{1}$ & $\mathrm{~B}_{2}$ & $\mathrm{~B}_{3}$ & $\mathrm{~B}_{4}$ & $\mathrm{~B}_{5}$ & Merged \\
\hline $\mathrm{DC}$ & 75.15 & 80.31 & 83.64 & 81.02 & 85.66 & $\mathbf{8 6 . 3 8}$ \\
\hline $\mathrm{HD}$ & 2.15 & 1.95 & 1.77 & 1.90 & 1.68 & $\mathbf{1 . 6 6}$ \\
\hline AVD & 4.35 & 3.58 & 2.90 & 3.43 & 2.60 & $\mathbf{2 . 5 2}$ \\
\hline
\end{tabular}

Table 3: We compare the segmentation performance of the outputs from each block in SegNetMRI5 model without the $1 \times 1$ convolution and the segmentation output produced by the SegNetMRI ${ }_{5}$ via merging.

ure 5, along with their corresponding residuals. (We set the error ranges from [ $\left[\begin{array}{ll}0 & 0.1\end{array}\right]$ on a $\left[\begin{array}{ll}0 & 1\end{array}\right]$ pre-scaled image.) We find that SegNetMRI achieves the minimal reconstruction error, especially in the meaningful tissue regions. We also give averaged reconstruction performance measures in Table 2 using peak signal-to-noise ratio (PSNR) and the corresponding normalized mean squared error (NMSE) on all 37 test MRI.

Discussion It is worth noting that the the model in [Liu et al., 2017 achieves better segmentation performance than the $\mathrm{MRN}_{5}$ model, but the reconstruction quality is worse than $\mathrm{MRN}_{5}$ both qualitatively and quantitatively. The original work in [Liu et al., 2017] is devote to joint natural image denoising and segmentation, while for medical image analysis, the absolute reconstruction and segmentation accuracy is equally important. Usually, segmented MRI will also be provided by radiologists for diagnosis. A reconstruction error can thus cause the loss of valuable diagnostic information. In contrast, the SegNetMRI model achieves better performance on both reconstruction and segmentation.

In SegNetMRI ${ }_{N}$, the output of $N$ MSN decoders are concatenated and merged into the final segmentation using a $1 \times 1$ convolution. The ensemble learning can make full use of the information from different depth of the SegNetMRI and produce better segmentation accuracy. We take the SegNetMRI ${ }_{5}$ on the gray matter tissue of all the test MRI data for example. In Table 3, we show the segmentation performance of the outputs from each block in SegNetMRI ${ }_{5}$ model without the $1 \times 1$ convolution, and we compare them with the segmentation output produced after merging the SegNetMRI ${ }_{5}$ outputs with $1 \times 1$ convolution. The output of SegNetMRI ${ }_{5}$ achieves better segmentation performance, showing the effectiveness of this ensemble structure.

In Figure 6 , we show the segmentation accuracy (in Dice Coefficient metric) as a function of blocks, $N$. The reconstruction quality (in PSNR metric) improves as the number of the blocks increases in the SegNetMRI model, but at the expense of longer training time.

\section{Conclusion}

Automatic segmentation of MRI is an important problem in medical imaging, and with the recent adoption of CS-MRI by

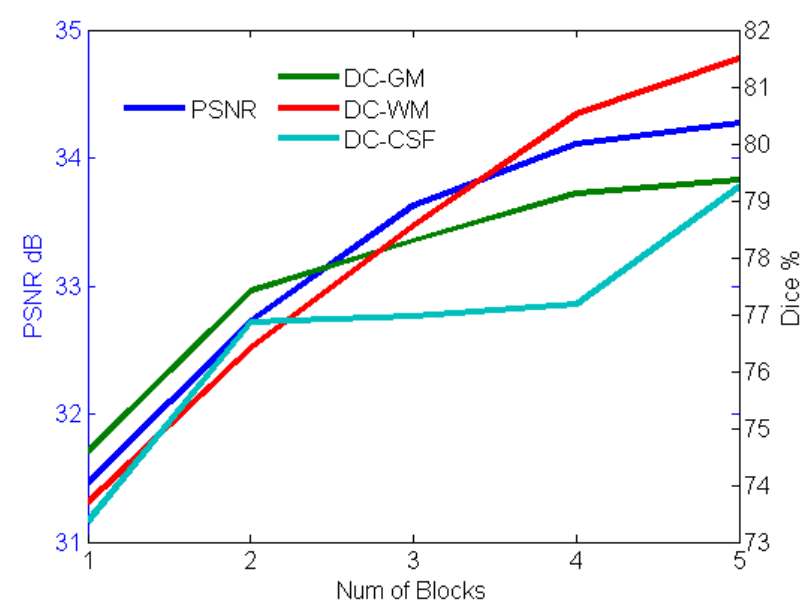

Figure 6: The model performance of SegNetMRI ${ }_{N}$ architecture as a function of the number of blocks.

industry, segmentation techniques that take CS-MRI reconstruction into account are needed. After verifying that the two tasks suffer when done independently, we proposed a deep neural network architecture called SegNetMRI to merge the MRI reconstruction and segmentation problems into a joint framework. Our experiments show that doing simultaneous reconstruction and segmentation can positively reinforce each other, improving both tasks significantly.

\section{Acknowledgement}

This work was supported in part by the National Natural Science Foundation of China under Grants 61571382, 81671766, 61571005, 81671674, U1605252, 61671309 in part by the Guangdong Natural Science Foundation under Grant 2015A030313007, in part by the Fundamental Research Funds for the Central Universities under Grant 20720160075, 20720180059, in part by the National Natural Science Foundation of Fujian Province, China under Grant 2017J01126. (Corresponding author: Xinghao Ding)

\section{References}

[Aljabar et al., 2009] Paul Aljabar, Rolf A Heckemann, Alexander Hammers, Joseph V Hajnal, and Daniel Rueckert. Multi-atlas based segmentation of brain images: atlas selection and its effect on accuracy. Neuroimage, 46(3):726-738, 2009.

[Artaechevarria et al., 2009] Xabier Artaechevarria, Arrate Munoz-Barrutia, and Carlos Ortiz-de Solórzano. Combination strategies in multi-atlas image segmentation: Application to brain MR data. IEEE Trans. on Medical Imaging, 28(8):1266-1277, 2009.

[Atkinson et al., 1999] David Atkinson, Derek LG Hill, Peter NR Stoyle, Paul E Summers, Stuart Clare, Richard Bowtell, and Stephen F Keevil. Automatic compensation of motion artifacts in MRI. Magnetic Resonance in Medicine, 41(1):163-170, 1999. 
[Bahrami et al., 2017] Khosro Bahrami, Islem Rekik, Feng Shi, and Dinggang Shen. Joint reconstruction and segmentation of 7t-like $\mathrm{mr}$ images from $3 \mathrm{t}$ mri based on cascaded convolutional neural networks. In MICCAI, pages 764772. Springer, 2017.

[Candès et al., 2006] Emmanuel J Candès, Justin Romberg, and Terence Tao. Robust uncertainty principles: Exact signal reconstruction from highly incomplete frequency information. IEEE Trans. on Information Theory, 52(2):489-509, 2006.

[Chen et al., 2017] Hao Chen, Qi Dou, Lequan Yu, Jing Qin, and Pheng-Ann Heng. Voxresnet: Deep voxelwise residual networks for brain segmentation from 3D MR images. NeuroImage, 2017.

[Çiçek et al., 2016] Özgün Çiçek, Ahmed Abdulkadir, Soeren S Lienkamp, Thomas Brox, and Olaf Ronneberger. 3D U-Net: learning dense volumetric segmentation from sparse annotation. In MICCAI, pages 424-432. Springer, 2016.

[Dong et al., 2017] Hao Dong, Guang Yang, Fangde Liu, Yuanhan Mo, and Yike Guo. Automatic brain tumor detection and segmentation using u-net based fully convolutional networks. In Annual Conference on Medical Image Understanding and Analysis, pages 506-517. Springer, 2017.

[Donoho, 2006] David L Donoho. Compressed sensing. IEEE Trans. on Information Theory, 52(4):1289-1306, 2006.

[Fessler, 2017] Jeffrey A Fessler. Medical image reconstruction: a brief overview of past milestones and future directions. arXiv preprint arXiv:1707.05927, 2017.

[Hricak and Amparo, 1984] H Hricak and EG Amparo. Body MRI: alleviation of claustrophobia by prone positioning. Radiology, 152(3):819-819, 1984.

[Huang et al., 2014] Yue Huang, John Paisley, Qin Lin, Xinghao Ding, Xueyang $\mathrm{Fu}$, and Xiao-Ping Zhang. Bayesian nonparametric dictionary learning for compressed sensing MRI. IEEE Trans. on Image Processing, 23(12):5007-5019, 2014.

[Lai et al., 2016] Zongying Lai, Xiaobo Qu, Yunsong Liu, Di Guo, Jing Ye, Zhifang Zhan, and Zhong Chen. Image reconstruction of compressed sensing MRI using graphbased redundant wavelet transform. Medical Image Analysis, 27:93-104, 2016.

[Lee et al., 2017] Dongwook Lee, Jaejun Yoo, and Jong Chul Ye. Deep residual learning for compressed sensing MRI. In ISBI, pages 15-18. IEEE, 2017.

[Li et al., 2017] Boyi Li, Xiulian Peng, Zhangyang Wang, Jizheng $\mathrm{Xu}$, and Dan Feng. Aod-net: All-in-one dehazing network. In ICCV, Oct 2017.

[Liu et al., 2017] Ding Liu, Bihan Wen, Xianming Liu, and Thomas S Huang. When image denoising meets high-level vision tasks: A deep learning approach. arXiv preprint arXiv:1706.04284, 2017.
[Lustig et al., 2007] Michael Lustig, David Donoho, and John M Pauly. Sparse MRI: The application of compressed sensing for rapid MR imaging. Magnetic Resonance in Medicine, 58(6):1182-1195, 2007.

[Mendrik et al., 2015] Adriënne M Mendrik, Koen L Vincken, Hugo J Kuijf, Marcel Breeuwer, Willem $\mathrm{H}$ Bouvy, Jeroen De Bresser, Amir Alansary, Marleen De Bruijne, Aaron Carass, Ayman El-Baz, et al. MRBrainS challenge: online evaluation framework for brain image segmentation in 3T MRI scans. Computational Intelligence and Neuroscience, 2015:1, 2015.

[Moeskops et al., 2016] Pim Moeskops, Max A Viergever, Adriënne M Mendrik, Linda S de Vries, Manon JNL Benders, and Ivana Išgum. Automatic segmentation of MR brain images with a convolutional neural network. IEEE Trans. on Medical Imaging, 35(5):1252-1261, 2016.

[Nie et al., 2016] Dong Nie, Li Wang, Yaozong Gao, and Dinggang Sken. Fully convolutional networks for multimodality isointense infant brain image segmentation. In ISBI, pages 1342-1345. IEEE, 2016.

[Qu et al., 2014] Xiaobo Qu, Yingkun Hou, Fan Lam, Di Guo, Jianhui Zhong, and Zhong Chen. Magnetic resonance image reconstruction from undersampled measurements using a patch-based nonlocal operator. Medical Image Analysis, 18(6):843-856, 2014.

[Ravishankar and Bresler, 2011] Saiprasad Ravishankar and Yoram Bresler. MR image reconstruction from highly undersampled k-space data by dictionary learning. IEEE Trans. on Medical Imaging, 30(5):1028-1041, 2011.

[Ronneberger et al., 2015] Olaf Ronneberger, Philipp Fischer, and Thomas Brox. U-net: Convolutional networks for biomedical image segmentation. In MICCAI, pages 234-241. Springer, 2015.

[Schlemper et al., 2017] Jo Schlemper, Jose Caballero, Joseph V Hajnal, Anthony Price, and Daniel Rueckert. A deep cascade of convolutional neural networks for MR image reconstruction. In IPMI, pages 647-658. Springer, 2017.

[Steenwijk et al., 2013] Martijn D Steenwijk, Petra JW Pouwels, Marita Daams, Jan Willem van Dalen, Matthan WA Caan, Edo Richard, Frederik Barkhof, and Hugo Vrenken. Accurate white matter lesion segmentation by $\mathrm{k}$ nearest neighbor classification with tissue type priors (kNN-TTPs). NeuroImage: Clinical, 3:462-469, 2013.

[Stollenga et al., 2015] Marijn F Stollenga, Wonmin Byeon, Marcus Liwicki, and Juergen Schmidhuber. Parallel multidimensional lstm, with application to fast biomedical volumetric image segmentation. In NIPS, pages 2998-3006, 2015.

[Wang et al., 2015] Li Wang, Yaozong Gao, Feng Shi, Gang Li, John H Gilmore, Weili Lin, and Dinggang Shen. Links: Learning-based multi-source integration framework for segmentation of infant brain images. NeuroImage, 108:160-172, 2015. 
[Wang et al., 2016] Shanshan Wang, Zhenghang Su, Leslie Ying, Xi Peng, Shun Zhu, Feng Liang, Dagan Feng, and Dong Liang. Accelerating magnetic resonance imaging via deep learning. In ISBI, pages 514-517. IEEE, 2016.

[Zhang et al., 2011] Haichao Zhang, Jianchao Yang, Yanning Zhang, Nasser M Nasrabadi, and Thomas S Huang. Close the loop: Joint blind image restoration and recognition with sparse representation prior. In $I C C V$, pages 770-777. IEEE, 2011. 